\title{
Ubiquitination in the rice blast fungus Magnaporthe oryzae: from development and pathogenicity to stress responses
}

\author{
Yu Wang, Nan Yang, Yunna Zheng, Jiaolin Yue, Vijai Bhadauria, You-Liang Peng and Qian Chen* (1)
}

\begin{abstract}
Ubiquitination is a vital protein post-translational modification (PTM) prevalent in eukaryotes. This modification regulates multiple cellular processes through protein degradation mediated by the $26 \mathrm{~S}$ proteasome or affecting protein-protein interaction and protein localization. Magnaporthe oryzae causes rice blast disease, which is one of the most devastating crop diseases worldwide. In M. oryzae, ubiquitination plays important roles in growth, pathogenicity, stress response and effector-mediated plant-pathogen interaction. In this review, we summarize the roles of ubiquitination components in the above biological processes of M. oryzae, including single- or multi-subunit E3s, E2s, components of 265 proteasome and also deubiquitinating enzymes. The essential function of ubiquitination in plantfungus interaction is also discussed. Moreover, this review presents several issues related to the ubiquitination system in M. oryzae, which need to be further explored in future researches.
\end{abstract}

Keywords: Ubiquitination, Pathogenicity, Stress response, Magnaporthe oryzae

\section{Background}

In eukaryotes, ubiquitination is an important post-translational modification (PTM) that contributes to various cellular processes, including growth and biotic or abiotic stress responses. Ubiquitination is usually involved in these cellular processes through regulating protein stability, protein activity or protein-protein interaction. Protein ubiquitination proceeds via three sequential enzymatic reactions. The first step is the activation of the 76-amino acid ubiquitin protein by the ubiquitinactivating enzyme (E1) in an ATP-dependent manner. The activated ubiquitin is then transferred to an active cysteine residue of a ubiquitin-conjugating enzyme (E2) to form the E2-ubiquitin intermediate. Finally, a particular ubiquitin ligase (E3) recruits the targeted substrate

*Correspondence: qianchen@cau.edu.cn

MOA Key Lab of Pest Monitoring and Green Management, Department of Plant Pathology, College of Plant Protection, China Agricultural University, Beijing 100193, China protein, and catalyzes the transfer of ubiquitin from E2 to the substrate with the formation of an isopeptide bond between the carboxy-terminal glycine of ubiquitin and a lysine residue of the target protein (Callis 2014).

Typically, E3 ubiquitin ligases are responsible for the specific recognition of target proteins. E3 ubiquitin ligases are categorized as single-subunit E3 ligases, including homologous to E6-AP carboxyl terminus (HECT), really interesting new gene (RING) and U-box, and multi-subunit E3 ligases cullin (Cul)-RING ligases (CRLs) (Santner and Estelle 2010). Four classes of CRLs are further divided as sphase kinase-associated protein1Cul1-F-box ligases (SCFs), bricabrac-tramtrak-broad complex (BTB), DNA damage-binding protein (DDB) and anaphase-promoting complex (APC) (Hua and Vierstra 2011; Yu et al. 2016). Take the SCF complex as an example, multi-subunit E3 ligase is assembled through a conserved scaffold CUL1, which further recruits a RING protein RBX1 (directs E2 to the SCF complex) and SKP1/ F-box proteins (work as substrate-targeting subunit) original author(s) and the source, provide a link to the Creative Commons licence, and indicate if changes were made. The images or other third party material in this article are included in the article's Creative Commons licence, unless indicated otherwise in a credit line to the material. If material is not included in the article's Creative Commons licence and your intended use is not permitted by statutory regulation or exceeds the permitted use, you will need to obtain permission directly from the copyright holder. To view a copy of this licence, visit http://creativecommons.org/licenses/by/4.0/. 
(Cardozo and Pagano 2004). SKP1 mediates the linkage between F-box and CUL1, and this is vital for stabilizing the SCF complex (Hua and Vierstra 2011).

Generally, E3 ligase recruits substrates directly, while E2 works by binding to E3 (Ye and Rape 2009). In recent years, however, it is reported that in addition to acting as an intermediate protein, E2 also directly binds to target proteins and contributes to determining the specificity of substrate recognition. For example, PHO2, a ubiquitinconjugating enzyme in Arabidopsis, directly associates with the phosphate transporter PHO1 and negatively regulates its stability (Liu et al. 2012). Furthermore, UBC27 and UBC32 directly interact with their target proteins $\mathrm{ABI} 1$ and PIP2;1/PIP2;2 respectively, and promote the ubiquitination and thereby degradation of target proteins (Pan et al. 2020; Chen et al. 2021). These findings shed light on at least some of the ubiquitin-conjugating enzymes that participate in target recognition directly (Fig. 1).

E2 or E2-E3 complex also determines the synthesis of polyubiquitin chains. Polyubiquitin chains, including
K11-, K27-, K48- and K63-linked chains, are produced according to different lysine connections between the two adjacent ubiquitins. K48-linked chains are often considered to function in proteasome-mediated protein degradation. However, in the past decade, scientists have found that all non-K63 linkages could target proteins for degradation (Xu et al. 2009). The synthesis of a particular polyubiquitin chain is determined by specific E2s or E2-E3 complexes. For example, Ubc6p, an E2 involved in yeast ERAD, primarily synthesizes K11 linkages (Xu et al. 2009); the Mms2/Ubc13 complex preferentially catalyzes the K63-linked chains (Eddins et al. 2006). Besides linking to the lysine residue in target proteins, ubiquitin chains could link onto other amino acids, including cysteine (form thioester bond with glycine of ubiquitin), serine/ threonine (form oxyester bond with glycine of ubiquitin) and $\mathrm{N}$-terminal (form a peptide bond with glycine of ubiquitin) of substrate proteins (Cadwell and Coscoy 2005; Wang et al. 2007; Shimizu et al. 2010). Although the function of ubiquitin chains might be conservative, up to now, most of the related results are from yeast and

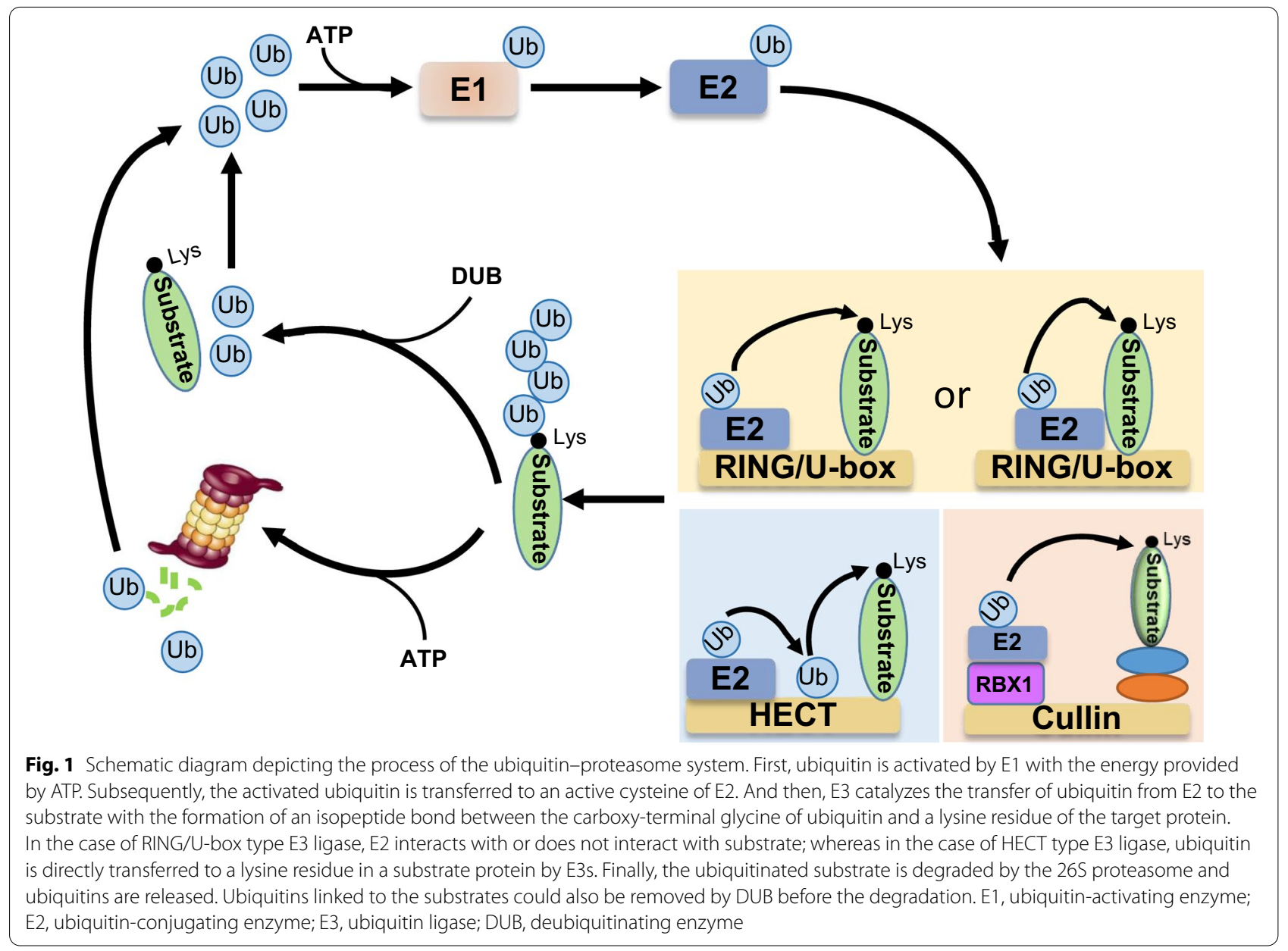


mammal, while the synthesis and function of polyubiquitin chains in pathogens are largely unclear.

Ubiquitination is a reversible course. Deubiquitinating enzymes (DUBs) contribute to removing ubiquitins from ubiquitinated proteins before their proteasomal degradation. As a result, DUB participates in different processes by inhibiting protein ubiquitination and subsequent degradation. Ubiquitin-specific proteases (UBPs) are the largest family of DUBs. There are 16 UBP proteins in Saccharomyces cerevisiae (Wilkinson 1997) and 27 UBP proteins in Arabidopsis thaliana (Liu et al. 2008). Take Arabidopsis UBP12/UBP13 as an example, UBP12 and $U B P 13$ work redundantly in the negative regulation of plant innate immune responses (Ewan et al. 2011). However, UBP12/UBP13 associates with the transcription factor MYC2 in the nucleus and enhances JA response by stabilizing MYC2 (Jeong et al. 2017). ROOT MERISTEM GROWTH FACTOR (RGF) 1 is an important peptide hormone that modifies root growth. UBP12/UBP13 contributes to maintaining the root cell sensitivity to RGF1 through counteracting the ubiquitination and degradation of the RGF1 receptor (An et al. 2018). In the process of nitrogen deficiency-induced leaf senescence, UBP12/ UBP13 counteracts the function of E3 ligase Nitrogen Limitation Adaptation (NLA) to increase the stability of the transcription factor ORESARA1 (ORE1) (Park et al. 2019). Biochemical and genetic investigation results have demonstrated that deubiquitinating enzymes UBP12 and UBP13 participate in various signaling pathways by reducing the ubiquitination and degradation of different targets. More detailed knowledge about DUBs can be found in excellent reviews such as that by Komander et al. (2009) and by Mevissen and Komander (2017).

Ubiquitin-like proteins (Ubls), including small ubiquitin-related modifier (SUMO), neural precursor cellexpressed developmentally down-regulated 8 (NEDD8) and ubiquitin-related modifier 1 (URM1), are also uncovered in eukaryotes (Hochstrasser 2000). Among them, SUMOylation is the best-studied Ubl modification. SUMOylation is also catalyzed by E1, E2 and E3, and attaches the $\mathrm{C}$ terminus of SUMO to the lysine residue of target proteins. Compared with ubiquitination, the relatively smaller SUMO system consists of a single E1, a single E2 and several E3s, although it plays comprehensive roles in the development and stress responses of eukaryotes. SUMOylation usually works through affecting protein stability (generally improve protein stability), protein localization (such as nuclear-cytosolic transport) and protein interaction (Miura et al. 2007).

Magnaporthe oryzae is a hemibiotrophic fungus that causes rice blast, one of the most destructive rice diseases worldwide (Wilson and Talbot 2009). M. oryzae grows as branching hyphae and produces three-celled conidia on the top of the conidiophore (Wilson 2021). The first step of its infection process is to form appressorium after adhesion of conidia to the plant surface. Subsequently, a penetration peg is formed to pierce through host surface with the help of the elevated appressorial turgor pressure. Upon successful invasion of plant cells, invasive hyphae (IH) are established (Talbot 2003; Gilbert et al. 2006). Defects in any step of the infection process could lead to the reduction or loss of pathogenicity at last.

In $M$. oryzae, there are 8 genes encoding ubiquitinactivating enzymes including ubiquitin-like activating enzymes, 21 genes encoding ubiquitin-conjugating enzyme and 94 genes encoding E3 ligase, according to the protein-similarity search result using InterPro domain (Oh et al. 2012). However, most of these predicted genes in the ubiquitin system have not been verified until now. The microarray data shows that more predicted genes in the ubiquitin system (39\%) are induced during conidia germination than the entire transcriptome (21\%) (Oh et al. 2012), which implies that some proteins in conidia germination are involved in ubiquitination process. In addition, inhibition of ubiquitin-mediated protein degradation using the $26 \mathrm{~S}$ proteasome inhibitor remarkably attenuates the invasion process in a dose-dependent manner, from conidia germination, appressorium formation to the pathogenicity of M. oryzae (Oh et al. 2012). This result demonstrates that the ubiquitin system also plays important roles in multiple biological processes of M. oryzae. Although more and more key proteins in the ubiquitin-proteasome system have been characterized in recent years, here we mainly present recent advances on the biological functions of ubiquitination in the development, pathogenicity and stress responses of $M$. oryzae and also in $M$. oryzae-rice interaction.

\section{Roles of the ubiquitin-proteasome system (UPS) in growth and pathogenicity of $M$. oryzae E3 ligase}

The SCF complex is a kind of multi-subunit E3 ligase. In M. oryzae, MoGrr1 is the first reported F-box encoding gene, a core component of the SCF complex. Grr1 is originally identified in yeast, and MoGrr1 can complement the growth defect of grr 1 mutant in yeast (Oh et al. 2012). Targeted disruption of MoGrr1 results in abnormal vegetative growth, conidial and melanin production, and subsequently attenuates the virulence of $M$. oryzae to host plants. Complementation experiments exhibit that the full-length MoGrrl, but not the deletion of the F-box domain, can recover the pathogenicity of $\Delta M o g r r 1$. In detail, transformants that overexpress the F-box domaindeleted MoGrr1 (MoGrr1 $\Delta$ F-box) in the $\Delta$ Mogrr1 mutant background exhibit a complete loss of conidiation and reduced virulence, similar to the phenotypes 
observed in the $\Delta$ Mogrr1 mutant strain. Therefore, the whole function of MoGrr1 is required for the development and pathogenicity of $M$. oryzae (Oh et al. 2012).

Recently, a study reported that MoGrr1 and another HECT type E3 ligase Ptr1 negatively regulate the basal protein level of sirtuin 2 (Sir2), an antioxidation regulator required for suppressing the host oxidative burst, in the absence of oxidative stress. Sir2 directly interacts with MoGrr1, and the protein level of Sir2 is accumulated in the $\Delta$ Mogrr1 mutant, which is logical to be regarded as the substrate-E3 relationship ( $\mathrm{Li}$ et al. 2020). However, in the presence of oxidative stress, another HECT type E3 ligase Upl3, which is also critical to the virulence of M. oryzae, helps maintain Sir2 protein accumulation ( $\mathrm{Li}$ et al. 2020). In addition, the author proved that all these three E3 ligases function at the post-translational level. Therefore, Upl3 might indirectly regulates the protein level of Sir2. Taken together, the differential regulation of Sir2 by multiple E3 ligases provides evidence that distinct E3 ligases can antagonistically regulate a single protein at different environmental conditions.

Frp1, an F-box protein in Fusarium oxysporum, is pivotal to the pathogenicity of $F$. oxysporum on tomato (Duyvesteijn et al. 2005). The homologous of Frp1 is identified as MoFrp1 in M. oryzae. MoFrp1 interacts with MoSkp1, the scaffold protein in the SCF complex (Prakash et al. 2016). In RNAi-based MoSkp1 knockdown transformants, abnormal conidiogenesis-related phenotypes are observed, such as reduced sporulation, small conidium, delayed spore germination, and the inability to form appressoria. In addition, in $\Delta M o s k p 1$ mutant, the ubiquitination level of total protein is significantly reduced, which is consistent with the ubiquitination function of the SCF complex (Prakash et al. 2016). Based on these results, we can conjecture that the SCF complex might function in the growth and virulence of $M$. oryzae through mediating the ubiquitination of key regulators in these pathways. However, few substrates have been identified in M. oryzae so far.

To identify more F-box proteins that are involved in the pathogenicity of M. oryzae, Shi et al. (2019) screened 24 F-box candidate proteins that interacted with MoSkp1. Finally, 17 F-box proteins were confirmed to interact with MoSkp1, exhibiting the potential to form an SCF complex. In the aspect of phenotype, the author found that the mutants of 21 F-box protein-coding genes showed no difference in virulence compared with wild type. Combined together, three F-box proteins, including MoFwd1, MoCdc4 and MoFbx15, could interact with MoSkp1 and are required for the development and virulence of $M$. oryzae. MoFbx15 and MoCdc4 are distributed in the nucleus, while MoFwd1 is localized to the cytosol. MoFwd1 and MoCdc4 interact with MoSkp1 through the
F-box domain, which is consistent with the interaction characteristics of the SCF complex. Phenotypic analysis showed that mutants of MoFwd1, MoCdc4 and MoFbx15 cause virulence defects, partially due to delayed conidia germination and reduced appressorial formation (Shi et al. 2019). MoFwd1 is also involved in circadian rhythm through modifying the transcription and protein stability of MoFrq, a core circadian regulator (Shi et al. 2019). Recent studies showed that the ubiquitination level of total protein in $\Delta M o f b x 15$ is decreased compared with that of wild type, which further proved that SCF ubiquitin ligases containing F-box proteins play indispensable roles in the development and pathogenicity of $M$. oryzae by affecting protein ubiquitination (Lim and Lee 2020).

Single-subunit E3 ligases, especially RING-type E3 ligases, have also been identified in M. oryzae. Hrd1 is a key E3 ligase of the endoplasmic reticulum-associated degradation (ERAD) pathway, and MoHrd1 is identified in M. oryzae. IP-MS data to separate putative interacting proteins of MoHrd1 revealed that MoHrd1 is involved in the secretory pathway, energy synthesis and metabolism (Jiang et al. 2018). Cell biological and biochemical investigation results showed that both MoHrd1 and MoDer1 have conserved functions in the ERAD system, including ER localization, ERAD substrate degradation and the induction of unfolded protein response (UPR) in their knockout mutants. With regard to biological function, MoHrd1 and MoDer1 are involved in the growth, protein secretion and pathogenicity of $M$. oryzae (Tang et al. 2020). The studies of MoHrd1 shed light on the essential role of ERAD in the development and virulence of $M$. oryzae. However, only a few proteins in the ERAD pathway have been identified so far, and the regulation mechanism of the ERAD system in the development and pathogenicity of $M$. oryzae is largely unknown. Other RING-type E3 ligases, including MoBre1, MoRad18 and MoUbr1, have also been characterized in M. oryzae (Shi et al. 2016). Although the function of Bre1, Rad18 and Ubr1 in mammals and yeast are clearly uncovered, the specific substrates of these E3s have not been identified in the regulation pathway of $M$. oryzae.

\section{E2 and other components in the ubiquitin-proteasome system}

E2-conjugating enzyme, as another important component in the ubiquitination pathway, plays an important role in cell signaling by influencing protein stability. There are 21 E2 enzymes in $M$. oryzae, but the corresponding studies are lacking (Oh et al. 2012). MoRad6 is an identified ubiquitin-conjugating enzyme and is pivotal to the growth and development of $M$. oryzae (Shi et al. 2016). The disruption of MoRad6 leads to defects in growth, sporulation, conidial germination, appressorium 
formation and infection of host plant. Similar to its homolog Rad6 in yeast and mammal, MoRad6 interacts with three RING-type E3 ligases, MoBre1, MoRad18 and MoUbr1, in M. oryzae. In terms of fungal growth, conidial adhesion, germination and appressorium formation, MoRad6 functions mainly by interacting with MoUbr1, which is involved in protein degradation through the $\mathrm{N}$-end rule pathway (Shi et al. 2016). The study of MoRad6 suggests that E2-conjugating enzymes are also essential to both the development and pathogenicity of M. oryzae. Thus, more regulation mechanisms of E2 need to be uncovered in the future.

Other important components in the ubiquitin-proteasome system are also identified. Targeted deletion of MGG_01282, a polyubiquitin-encoding gene, resulted in a significant reduction in the ubiquitination level of total protein (Oh et al. 2012). This gene deletion leads to pleiotropic effects on $M$. oryzae, including development and the whole invasion process. Compared with the wild-type strain, the MGG_01282-deleted mutant presents significant changes in fungal growth, morphology and development. The growth inhibition is more severe under nitrogen starvation treatment. As to the effect on pathogenicity, MGG_01282 mutant shows reduced pathogenicity on barely (Oh et al. 2012). The result on phenotype analysis of the MGG_01282-deleted mutant further supports that ubiquitin is essential to a lot of biological processes in $M$. oryzae, including those associated with vegetative growth, stress responses and pathogenicity.

Deubiquitination is an indispensable part of the ubiquitin-mediated proteasome degradation system. In M. ory$z a e$, the role of deubiquitinating enzymes in development and pathogenicity is also characterized. Deubiquitinating enzyme MoUbp14 is homologous to Ubp14 in S. cerevisiae. Deletion of Ubp14 in yeast results in the accumulation of free polyubiquitin chains, in line with the expected function of the deubiquitinating enzyme (Wang et al. 2018). Targeted deletion of MoUbp14 in M. oryzae results in reduced sporulation, irregular appressorium formation and decreased pathogenicity on the host. According to the pull-down assay result, some proteins involved in carbohydrate metabolism and stress responses, and two key rate-limiting enzymes in gluconeogenesis, MoFbp1 and MoPck1, are identified as the Ubp14-interacting proteins (Wang et al. 2018). The ubiquitination levels of MoFbp1 and MoPck1 are increased in the $\Delta$ Moubp 14 mutant; however, the protein accumulation of MoFbp1 and MoPck1 is also elevated in the $\Delta$ Moubp14 mutant. The author hypothesized that MoUbp14 is responsible for removing ubiquitins from ubiquitinated proteins, and it can help in providing sufficient ubiquitin for the degradation of MoFbp1 and MoPck1 (Wang et al. 2018). It is also possible that the substrates of MoUbp14 are not so specific. For example, the E3 ligase of MoFbp1 and MoPck1 might also be the substrate of MoUbp14. The final comprehensive effect of MoUbp14 on MoFbp1 and MoPck1 is a reduction in their ubiquitination level but a promotion of their degradation.

MoUbp4 and MoUbp8 are another two deubiquitinating enzymes involved in the growth and pathogenicity of $M$. oryzae. Ubiquitination levels of total protein are increased in the MoLbp4- or MoUbp8-deleted mutant. These two genes are required for the growth and virulence of M. oryzae (Que et al. 2020; Yang et al. 2020). However, the studies of MoUbp4 and MoUbp 8 are focused on their biological functions analysis, and the underlying mechanism behind the phenotype is largely unknown.

As mentioned earlier, conidium germination and appressorium formation are delayed in $M$. oryzae by the proteasome inhibitor, and subsequently the virulence is decreased (Oh et al. 2012). The $20 \mathrm{~S}$ proteasome, as a subunit of the $26 \mathrm{~S}$ proteasome, is important to the pathogenicity of the $M$. oryzae race KJ301 by inhibition of protein turnover (Wang et al. 2011). According to the above summary, almost all published genes in ubiquitin-mediated pathways, from ubiquitination to degradation, play a vital role in regulation of the growth and pathogenicity of $M$. oryzae.

\section{Ubiquitin-like modification in M. oryzae}

Besides the ubiquitin pathway, the function of ubiquitinlike proteins in $M$. oryzae is also uncovered. SUMOylation is involved in a variety of signaling pathways and ultimately affects multiple phenotypes of plants and animals. Some components of the SUMO pathway have been identified in $M$. oryzae, including SUMO Smt3, E1-activating enzymes Aos1 and Uba2, the E2 Ubc9 and the E3 ligase Siz1 (Liu et al. 2018). In M. oryzae, deletion of genes in the SUMOylation pathway results in multiple defect phenotypes throughout almost all life cycle stages, such as reduced growth and sporulation, abnormal conidium and appressorium, change in pathogenicity as well as cell-cycle-related phenotypes. Through analysis of the SUMO proteome of M. oryzae, many pathogenicityrelated proteins, including those in the mitogen-activated protein kinase (MAPK) signaling pathway, UPR regulated proteins and cell-wall-related proteins, are identified as SUMOylation substrates (Liu et al. 2018). In addition, both cytoplasmic and apoplastic effector proteins cannot be well secreted in SUMO mutants. The above results prove the importance of the SUMO pathway in the development, stress responses and pathogenicity of $M$. oryzae (Liu et al. 2018).

The septin ring at the appressorium pore plays an essential role in the process of penetrating the leaf 
surface. It composes of four core septins, Sep3, Sep4, Sep5 and Sep6 (Dagdas et al. 2012). Liu et al. found that all these four septin proteins can be SUMOylated. Mutation of the SUMOylation site results in the mislocalization of septin proteins. The site-targeted mutant also exhibits less virulence compared with the wild-type strain. These results demonstrate that SUMOylation is necessary for the integrated function of serpin proteins (Liu et al. 2018).

In M. oryzae P131, MoUrm1 is a ubiquitin-like protein involved in vegetative growth and infection (Wang et al. 2019a). The $\Delta$ Mourm1 mutant exhibit a slight reduction in growth rate and an evident reduction in conidia formation. In addition, this mutant induces much smaller lesions compared with the wild-type and complemented strains (Wang et al. 2019a). Taken together, ubiquitinlike modifications are conserved in M. oryzae and play an important role in the development, stress responses and pathogenicity of $M$. oryzae.

\section{Ubiquitination is required for responses to different stresses in $M$. oryzae}

Various environment cues interfere with the growth and infection process of $M$. oryzae. Cell wall stress, osmotic stress and oxidative stress are majorly investigated in $M$. oryzae. The results of gene expression analysis showed that multiple genes associated with protein ubiquitination were differentially regulated during stress responses compared with non-treated control. Targeted disruption of MoGrr1 results in increased sensitivity to oxidative and cell wall stresses. The expression levels of several chitin synthase-encoding genes, CHS1, CHS6 and CHS7, are significantly decreased in $\Delta M o g r r 1$, which might be the reason why $\Delta$ Mogrr1 is sensitive to cell wall stresses (Guo et al. 2015). The integrated cell wall is essential to the growth and pathogenicity of $M$. oryzae. Mutants devoid of any one of several other ubiquitin-proteasome components in Table 1 also exhibited cell wall stress-sensitive phenotype (Table 1), implying ubiquitination might enhance the virulence of $M$. oryzae through the cell wall integrity (CWI) pathway.

Besides cell wall stress, ubiquitination also affects nitrogen starvation stress. MGG_01282 is highly induced during nitrogen starvation, leading to a significant elevation in protein ubiquitination level than wild type. By enriching polyubiquitinated proteins, 63 proteins (including several proteins associated with cytoskeleton and stress responses) were identified in the purified samples but not in the negative control (Oh et al. 2012). PUB4, another polyubiquitin-encoding gene, is also abundantly expressed during nitrogen-starvation stress and glucose starvation (McCafferty and Talbot 1998). Nitrogen starvation could induce autophagy; therefore, whether ubiquitination has crosstalk with autophagy in M. oryzae needs further exploration.

\section{Ubiquitination-mediated regulation of secreted effectors}

During infection, a pathogen secrets effectors into plant cells to interfere with host plant resistance by changing protein stability, localization or complex formation. Multiple studies showed that ubiquitination-mediated protein degradation play important role in pathogen-host interaction. For example, the effector AvrPtoB, an E3 ligase from Pseudomonas syringae, promotes the degradation of positive plant immunity proteins, including lectin RLK LecRK-IX.2 and EXO70B1, to enhance the Pto DC3000 virulence (Wang et al. 2019b; Xu et al. 2020). In addition, LecRK-IX.2 reversely phosphorylates AvrPtoB to reduce its E3 ligase activity (Xu et al. 2020). In M. ory$z a e$-rice interaction, the effector AvrPiz-t interacts with two RING-type E3 ligases, AVRPIZ-T INTERACTING PROTEIN 6 (APIP6) and APIP10, to suppress their ubiquitin ligase activities and promote their degradation. In return, both APIP6 and APIP10 can ubiquitinate AvrPiz$\mathrm{t}$ and subsequently degrade it (Park et al. 2012, 2016). Besides AvrPiz-t, APIP10 negatively regulates the stability of nucleotide-binding leucine-rich repeat (NB-LRR) resistance protein Piz- $t$ through the 26S-proteasome pathway. Thus, AvrPiz-t protects Piz-t via decreasing the E3 ligase activity of APIP10 during $M$. oryzae infection (Park et al. 2016). The above studies give us a complex but clear relationship among AvrPiz-t, APIP10 and Piz-t.

\section{Conclusions and future perspectives}

Over the past several years, many components in UPS and ubiquitin-like systems have been identified in $M$. oryzae. Ubiquitination plays pivotal roles in several biological processes, including conidium germination, appressorium formation and development, cell wall integrity, stress response and pathogenicity. However, compared with the detailed mechanism of ubiquitination that has been revealed in S. cerevisiae, a large number of component proteins in the ubiquitin system of M. oryzae, especially E3 ligases, are uncharacterized even at present. Moreover, the substrates of the characterized components remain elusive. Revealing the biological function of more E3/E2s and identifying their substrates in the future will help us to understand the underlying mechanism of ubiquitination in the pathogenicity of M. oryzae.

Although efficient methods to screen substrates of the ubiquitin system are still lacking, many substrates have been identified in yeast and model plant Arabidopsis via screening E3 ligase-interaction proteins or using PTM proteomics approaches. PTM proteomics approaches have been used to identify target proteins 
Table 1 Ubiquitin pathway-associated proteins in M. oryzae

\begin{tabular}{|c|c|c|c|c|c|}
\hline Type & Proteins & Encoding gene & Biological function & Targets & References \\
\hline \multirow[t]{4}{*}{ Polyubiquitin } & PUB4 & AF056625 & \multirow{3}{*}{$\begin{array}{l}\text { Induced/inhibited by environ- } \\
\text { mental stress }\end{array}$} & $?$ & \multirow[t]{3}{*}{ McCafferty and Talbot (1998) } \\
\hline & \multirow[t]{2}{*}{ UEP1/UEP3 } & AF056623 & & $?$ & \\
\hline & & AF056624 & & & \\
\hline & UN & MGG_01282 & $\begin{array}{l}\text { Growth, pathogenicity and } \\
\text { nitrogen-starvation stress }\end{array}$ & $?$ & Oh et al. (2012) \\
\hline E2 & MoRad6 & MGG_01756 & Growth and pathogenicity & $?$ & Shi et al. (2016) \\
\hline \multirow[t]{4}{*}{ RING type E3 ligase } & MoUbr1 & MGG_13171 & $\begin{array}{l}\text { Growth, pathogenicity and } \\
\mathrm{N} \text {-end protein degradation }\end{array}$ & $?$ & \\
\hline & MoBre1 & MGG_00139 & Growth and pathogenicity & $?$ & \\
\hline & MoRad18 & MGG_04175 & Growth and pathogenicity & $?$ & \\
\hline & MoHrd1 & MGG_09205 & $\begin{array}{l}\text { Appressorium formation, patho- } \\
\text { genicity and protein secretion }\end{array}$ & $?$ & $\begin{array}{l}\text { Jiang et al. (2018), Tang et al. } \\
\text { (2020) }\end{array}$ \\
\hline \multirow[t]{5}{*}{ SCF complex } & MoGrr1 & MGG_13065 & $\begin{array}{l}\text { Growth, cell wall integrity (CWI) } \\
\text { and other stress responses }\end{array}$ & $?$ & Guo et al. (2015), Li et al. (2020) \\
\hline & MoFwd1 & MGG_09696 & Growth and pathogenicity & MoFRQ & Shi et al. (2019) \\
\hline & MoCdc4 & MGG_08345 & Growth and pathogenicity & $?$ & \\
\hline & MoFbx15 & MGG_00768 & Growth and pathogenicity & $?$ & $\begin{array}{l}\text { Shi et al. (2019), Lim and Lee } \\
\text { (2020) }\end{array}$ \\
\hline & MoSkp1 & MGG_04978 & Growth, pathogenicity and CWI & $?$ & Prakash et al. (2016) \\
\hline \multirow[t]{3}{*}{ Deubiquitination enzyme } & MoUbp14 & MGG_08270 & $\begin{array}{l}\text { Growth, pathogenicity, CWI and } \\
\text { other stress responses }\end{array}$ & $?$ & Wang et al. (2018) \\
\hline & MoUbp4 & MGG_04957 & Growth, pathogenicity and CWI & $?$ & Que et al. (2020) \\
\hline & MoUbp8 & MGG_03527 & $\begin{array}{l}\text { Growth, pathogenicity, CWI, } \\
\text { other stress responses and } \\
\text { carbon catabolite repression }\end{array}$ & $?$ & Yang et al. (2020) \\
\hline \multirow[t]{2}{*}{ Proteasome component } & 205 proteasome & $?$ & Pathogenicity & $?$ & Wang et al. (2011) \\
\hline & $26 \mathrm{~S}$ proteasome & $?$ & Pathogenicity & $?$ & Oh et al. (2012) \\
\hline SUMO & Smt3 & MGG_05737 & $\begin{array}{l}\text { Growth, pathogenicity, CWI and } \\
\text { other stress responses }\end{array}$ & Multiple proteins & Liu et al. (2018) \\
\hline \multirow[t]{2}{*}{ SUMOE1 } & Aos 1 & MGG_01669 & $\begin{array}{l}\text { Growth, pathogenicity, CWI and } \\
\text { other stress responses }\end{array}$ & Multiple proteins & \\
\hline & Uba2 & MGG_06733 & $\begin{array}{l}\text { Growth, pathogenicity, CWI and } \\
\text { other stress responses }\end{array}$ & Multiple proteins & \\
\hline SUMO E2 & Ubc9 & MGG_00970 & $\begin{array}{l}\text { Growth, pathogenicity, CWI and } \\
\text { other stress responses }\end{array}$ & Multiple proteins & \\
\hline SUMO E3 & Sizl & MGG_08837 & $\begin{array}{l}\text { Growth, pathogenicity, CWI and } \\
\text { other stress responses }\end{array}$ & Multiple proteins & \\
\hline Ubiquitin-like modification & MoUrm1 & MGG_03978 & $\begin{array}{l}\text { Growth, pathogenicity, CWI and } \\
\text { other stress responses }\end{array}$ & MoAHP1 & Wang et al. (2019a, b) \\
\hline
\end{tabular}

of phosphorylation, acetylation and $N$-glycosylation in M. oryzae (Franck et al. 2015; Sun et al. 2017; Wang et al. 2017; Liang et al. 2018; Chen et al. 2020). In addition, the ubiquitination system has been studied well in yeast. Identification of the homologues of ubiquitination components and their substrates in yeast or other species gives us a clue to better understand the ubiquitin system in rice blast fungus. Based on these methods, we believe that more substrates of the ubiquitin system can be identified in the future to depth our understanding of ubiquitination in $M$. oryzae. Additionally, one protein can be ubiquitinated by different E3 ligases, and one E3 ligase can also target several proteins in response to different signals. Therefore, revealing the interaction rules of different E3s with their substrates is helpful for us to understand the complexity of ubiquitination in response to different signals.

Ubiquitination and other PTMs closely regulate each other in the field of plants and animals. For example, phosphorylation can directly modify the stability of proteins or regulate the activity of E3 liagese. Phosphorylated ABA transporter NRT1.2/NPF4.6 and aquaporin proteins PIP2;1/PIP2;2 exhibit a faster degradation rate, whereas the degradation of their non-phosphorylated forms is 
repressed (Chen et al. 2021; Zhang et al. 2021). Like ubiquitination, SUMOylation also occurs at certain lysine residues; however, it often acts in competition with ubiquitination to enhance the stability of its substrates. For example, SUMOylation of transcription factors MYB75, MYB30 and DREB2A inhibits the degradation of these proteins with different mechanisms (Catala et al. 2007; Zheng et al. 2012, 2020). Thus, the crosstalk of ubiquitination with other different types of PTMs is ubiquitous in eukaryotes. The underlying molecular mechanism may involve protein conformational changes, protein-protein interaction or competing for the same modified aminoacid sites. In $M$. oryzae, the deletion mutant in the E3 ligase-encoding gene, MoBre1, showed a reduced level of dimethylation and trimethylation of histone 3 lysine 4 (H3K4), implying that MoBre1 might be involved in the regulation of histone $\mathrm{H} 3$ methylation through monoubiquitination of histone $\mathrm{H} 2 \mathrm{~B}$, as reported in yeast (Lee et al. 2007; Shi et al. 2016). In addition, MoSkp1 contains multiple phosphorylation sites, suggesting that the activity or other aspects of the SCF complex might also be modified by phosphorylation (Prakash et al. 2016). Investigations on the mutual regulation between different PTMs will deepen our understanding of the complex function of PTMs.

\begin{abstract}
Abbreviations
APC: Anaphase-promoting complex; BTB: Bricabrac-tramtrak-broad complex; CRLs: Cullin (Cul)-RING ligases; CWI: Cell wall integrity; DDB: DNA damagebinding protein; DUBs: Deubiquitinating enzymes; ERAD: Endoplasmic reticulum-associated degradation; ETI: Effector-triggered immunity; ETN: Effector-triggered necrosis; HECT: Homologous to E6-AP carboxyl terminus; IH: Invasive hyphae; MAPK: Mitogen-activated protein kinase; NB-LRR: Nucleotidebinding leucine-rich repeat; NEDD8: Neural precursor cell-expressed developmentally down-regulated 8; PTI: Pathogen-associated molecular pattern-triggered immunity; PTM: Post-translational modification; RING: Really interesting new gene; SCF: Sphase kinase-associated protein 1-cul1-F-box ligases; SUMO: Small ubiquitin-related modifier; UBPs: Ubiquitin-specific proteases; URM1: Ubiquitin-related modifier 1 .
\end{abstract}

\section{Acknowledgements}

We thank the members in our lab for their advice on this manuscript.

\section{Authors' contributions}

QC and Y-LP designed the outline; YW and QC wrote the manuscript; $V B, N Y$, $Y Z$ and JY revised the manuscript. All authors read and approved the final manuscript.

\section{Funding}

This work was supported by the National Natural Science Foundation of China (32072368) and the 2115 Talent Development Program of China Agricultural University.

\section{Availability of data and materials}

Not applicable.

\section{Declarations}

Ethics approval and consent to participate Not applicable.
Consent for publication

Not applicable.

\section{Competing interests}

The authors declare that they have no competing interests.

Received: 25 October 2021 Accepted: 28 December 2021

Published online: 12 January 2022

\section{References}

An Z, Liu Y, Ou Y, Li J, Zhang B, Sun D, et al. Regulation of the stability of RGF 1 receptor by the ubiquitin-specific proteases UBP12/UBP13 is critical for root meristem maintenance. Proc Natl Acad Sci USA. 2018;115(5):1123-8. https://doi.org/10.1073/pnas.1714177115.

Cadwell K, Coscoy L. Ubiquitination on nonlysine residues by a viral E3 ubiquitin ligase. Science. 2005;309(5731):127-30. https://doi.org/10.1126/scien ce. 1110340.

Callis J. The ubiquitination machinery of the ubiquitin system. Arabidopsis Book. 2014;12: e0174. https://doi.org/10.1199/tab.0174.

Cardozo T, Pagano M. The SCF ubiquitin ligase: insights into a molecular machine. Nat Rev Mol Cell Biol. 2004;5(9):739-51. https://doi.org/10.1038/ nrm1471.

Catala R, Ouyang J, Abreu IA, Hu Y, Seo H, Zhang X, et al. The Arabidopsis E3 SUMO ligase SIZ1 regulates plant growth and drought responses. Plant Cell. 2007;19(9):2952-66. https://doi.org/10.1105/tpc.106.049981.

Chen XL, Liu C, Tang B, Ren Z, Wang GL, Liu W. Quantitative proteomics analysis reveals important roles of $\mathrm{N}$-glycosylation on ER quality control system for development and pathogenesis in Magnaporthe oryzae. PLoS Pathog. 2020;16(2): e1008355. https://doi.org/10.1371/journal.ppat.1008355.

Chen Q, Liu R, Wu Y, Wei S, Wang Q, Zheng Y, et al. ERAD-related E2 and E3 enzymes modulate the drought response by regulating the stability of PIP2 aquaporins. Plant Cell. 2021;33(8):2883-98. https://doi.org/10.1093/ plcell/koab141.

Dagdas YF, Yoshino K, Dagdas G, Ryder LS, Bielska E, Steinberg G, et al. Septinmediated plant cell invasion by the rice blast fungus, Magnaporthe oryzae. Science. 2012;336(6088):1590-5. https://doi.org/10.1126/science. 1222934.

Duyvesteijn RGE, van Wijk R, Boer Y, Rep M, Cornelissen BJC, Haring MA. Frp1 is a Fusarium oxysporum F-box protein required for pathogenicity on tomato. Mol Microbiol. 2005;57(4):1051-63. https://doi.org/10.1111/j. 1365-2958.2005.04751.x.

Eddins MJ, Carlile CM, Gomez KM, Pickart CM, Wolberger C. Mms2-Ubc13 covalently bound to ubiquitin reveals the structural basis of linkage-specific polyubiquitin chain formation. Nat Struct Mol Biol. 2006;13(10):91520. https://doi.org/10.1038/nsmb1148.

Ewan R, Pangestuti R, Thornber S, Craig A, Carr C, O'Donnell L, et al. Deubiquitinating enzymes AtUBP12 and AtUBP13 and their tobacco homologue NtUBP1 2 are negative regulators of plant immunity. New Phytol. 2011;191(1):92-106. https://doi.org/10.1111/j.1469-8137.2011.03672.x.

Franck WL, Gokce E, Randall SM, Oh Y, Eyre A, Muddiman DC, et al. Phosphoproteome analysis links protein phosphorylation to cellular remodeling and metabolic adaptation during Magnaporthe oryzae appressorium development. J Proteome Res. 2015;14(6):2408-24. https://doi.org/10. 1021/pr501064q.

Gilbert MJ, Thornton CR, Wakley GE, Talbot NJ. A P-type ATPase required for rice blast disease and induction of host resistance. Nature. 2006;440(7083):535-9. https://doi.org/10.1038/nature04567.

Guo M, Gao F, Zhu XL, Nie X, Pan YM, Gao ZM. MoGrr1, a novel F-box protein, is involved in conidiogenesis and cell wall integrity and is critical for the full virulence of Magnaporthe oryzae. Appl Microbiol Biot. 2015;99(19):807588. https://doi.org/10.1007/s00253-015-6820-x.

Hochstrasser M. Evolution and function of ubiquitin-like protein-conjugation systems. Nat Cell Biol. 2000;2(8):E153-7. https://doi.org/10.1038/35019 643.

Hua Z, Vierstra RD. The cullin-RING ubiquitin-protein ligases. Annu Rev Plant Biol. 2011;62:299-334. https://doi.org/10.1146/annurev-arpla nt-042809-112256.

Jeong JS, Jung C, Seo JS, Kim JK, Chua NH. The deubiquitinating enzymes UBP12 and UBP13 positively regulate MYC2 levels in jasmonate 
responses. Plant Cell. 2017;29(6):1406-24. https://doi.org/10.1105/tpc.17. 00216.

Jiang H, Lin L, Tang W, Chen X, Zheng Q, Huang J, et al. Putative interaction proteins of the ubiquitin ligase Hrd1 in Magnaporthe oryzae. Evol Bioinform Online. 2018;14:1176934318810990. https://doi.org/10.1177/11769 34318810990

Komander D, Clague MJ, Urbe S. Breaking the chains: structure and function of the deubiquitinases. Nat Rev Mol Cell Biol. 2009;10(8):550-63. https://doi. org/10.1038/nrm2731.

Lee JS, Shukla A, Schneider J, Swanson SK, Washburn MP, Florens L, et al. Histone crosstalk between $\mathrm{H} 2 \mathrm{~B}$ monoubiquitination and $\mathrm{H} 3$ methylation mediated by COMPASS. Cell. 2007;131(6):1084-96. https://doi.org/10. 1016/.j.cell.2007.09.046.

Li G, Qi X, Sun G, Rocha RO, Segal LM, Downey KS, et al. Terminating rice innate immunity induction requires a network of antagonistic and redoxresponsive E3 ubiquitin ligases targeting a fungal sirtuin. New Phytol. 2020;226(2):523-40. https://doi.org/10.1111/nph.16365.

Liang M, Zhang S, Dong L, Kou Y, Lin C, Dai W, et al. Label-Free quantitative proteomics of lysine acetylome identifies substrates of Gen5 in Magnaporthe oryzae autophagy and epigenetic regulation. mSystems. 2018;3(6): e00270-18. https://doi.org/10.1128/mSystems.00270-18.

Lim YJ, Lee YH. F-box only and CUE proteins are crucial ubiquitinationassociated components for conidiation and pathogenicity in the rice blast fungus, Magnaporthe oryzae. Fungal Genet Biol. 2020;144: 103473. https://doi.org/10.1016/j.fgb.2020.103473.

Liu Y, Wang F, Zhang H, He H, Ma L, Deng XW. Functional characterization of the Arabidopsis ubiquitin-specific protease gene family reveals specific role and redundancy of individual members in development. Plant J. 2008;55(5):844-56. https://doi.org/10.1111/j.1365-313X.2008.03557.x.

Liu TY, Huang TK, Tseng CY, Lai YS, Lin SI, Lin WY, et al. PHO2-dependent degradation of PHO1 modulates phosphate homeostasis in Arabidopsis. Plant Cell. 2012;24(5):2168-83. https://doi.org/10.1 105/tpc.112.096636.

Liu C, Li Z, Xing J, Yang J, Wang Z, Zhang H, et al. Global analysis of sumoylation function reveals novel insights into development and appressorium-mediated infection of the rice blast fungus. New Phytol. 2018;219(3):1031-47. https://doi.org/10.1111/nph.15141.

McCafferty HRK, Talbot NJ. Identification of three ubiquitin genes of the rice blast fungus Magnaporthe grisea, one of which is highly expressed during initial stages of plant colonisation. Curr Genet. 1998;33:352-61. https:// doi.org/10.1007/s002940050347.

Mevissen TET, Komander D. Mechanisms of deubiquitinase specificity and regulation. Annu Rev Biochem. 2017;86:159-92. https://doi.org/10.1146/ annurev-biochem-061516-044916.

Miura K, Jin JB, Hasegawa PM. Sumoylation, a post-translational regulatory process in plants. Curr Opin Plant Biol. 2007;10(5):495-502. https://doi. org/10.1016/j.pbi.2007.07.002.

Oh Y, Franck WL, Han SO, Shows A, Gokce E, Muddiman DC, et al. Polyubiquitin is required for growth, development and pathogenicity in the rice blast fungus Magnaporthe oryzae. PLoS ONE. 2012;7(8): e42868. https://doi. org/10.1371/journal.pone.0042868.

Pan W, Lin B, Yang X, Liu L, Xia R, Li J, et al. The UBC27-AIRP3 ubiquitination complex modulates ABA signaling by promoting the degradation of ABI1 in Arabidopsis. Proc Natl Acad Sci USA. 2020;1 17(44):27694-702. https:// doi.org/10.1073/pnas.2007366117.

Park CH, Chen S, Shirsekar G, Zhou B, Khang CH, Songkumarn P, et al. The Magnaporthe oryzae effector AvrPiz-t targets the RING E3 ubiquitin ligase APIP6 to suppress pathogen-associated molecular pattern-triggered immunity in rice. Plant Cell. 2012;24(11):4748-62. https://doi.org/10.1105/ tpc. 112.105429

Park CH, Shirsekar G, Bellizzi M, Chen S, Songkumarn P, Xie X, et al. The E3 ligase APIP10 connects the effector AvrPiz-t to the NLR receptor Piz-t in rice. PLoS Pathog. 2016;12(3): e1005529. https://doi.org/10.1371/journal.ppat. 1005529.

Park SH, Jeong JS, Seo JS, Park BS, Chua NH. Arabidopsis ubiquitin-specific proteases UBP12 and UBP13 shape ORE1 levels during leaf senescence induced by nitrogen deficiency. New Phytol. 2019;223(3):1447-60. https://doi.org/10.1111/nph.15879.

Prakash C, Manjrekar J, Chattoo BB. Skp1, a component of E3 ubiquitin ligase, is necessary for growth, sporulation, development and pathogenicity in rice blast fungus (Magnaporthe oryzae). Mol Plant Pathol. 2016;17(6):90319. https://doi.org/10.1111/mpp.12336.
Que YW, Xu Z, Wang CY, Lv WY, Yue XF, Xu L, et al. The putative deubiquitinating enzyme MoUbp4 is required for infection-related morphogenesis and pathogenicity in the rice blast fungus Magnaporthe oryzae. Curr Genet. 2020;66(3):561-76. https://doi.org/10.1007/s00294-019-01049-8.

Santner A, Estelle M. The ubiquitin-proteasome system regulates plant hormone signaling. Plant J. 2010;61(6):1029-40. https://doi.org/10.1111/j. 1365-313X.2010.04112.x.

Shi HB, Chen GQ, Chen YP, Dong B, Lu JP, Liu XH, et al. MoRad6-mediated ubiquitination pathways are essential for development and pathogenicity in Magnaporthe oryzae. Environ Microbiol. 2016;18(11):4170-87. https://doi. org/10.1111/1462-2920.13515.

Shi HB, Chen N, Zhu XM, Liang S, Li L, Wang JY, et al. F-box proteins MoFwd1, MoCdc4 and MoFbx15 regulate development and pathogenicity in the rice blast fungus Magnaporthe oryzae. Environ Microbiol. 2019;21(8):3027-45. https://doi.org/10.1111/1462-2920.14699.

Shimizu Y, Okuda-Shimizu Y, Hendershot LM. Ubiquitylation of an ERAD substrate occurs on multiple types of amino acids. Mol Cell. 2010;40(6):91726. https://doi.org/10.1016/j.molcel.2010.11.033.

Sun X, Li Z, Liu H, Yang J, Liang W, Peng YL, et al. Large-scale identification of lysine acetylated proteins in vegetative hyphae of the rice blast fungus. Sci Rep. 2017;7(1):15316. https://doi.org/10.1038/s41598-017-15655-4.

Talbot NJ. On the trail of a cereal killer: exploring the biology of Magnaporthe grisea. Annu Rev Microbiol. 2003;57:177-202. https://doi.org/10.1146/ annurev.micro.57.030502.090957.

Tang W, Jiang H, Aron O, Wang M, Wang X, Chen J, et al. Endoplasmic reticulum-associated degradation mediated by MoHrd1 and MoDer 1 is pivotal for appressorium development and pathogenicity of Magnaporthe oryzae. Environ Microbiol. 2020;22(12):4953-73. https://doi.org/10. 1111/1462-2920.15069.

Wang X, Herr RA, Chua WJ, Lybarger L, Wiertz EJ, Hansen TH. Ubiquitination of serine, threonine, or lysine residues on the cytoplasmic tail can induce ERAD of MHC-I by viral E3 ligase mK3. J Cell Biol. 2007;177(4):613-24. https://doi.org/10.1083/jcb.200611063.

Wang Y, Kim SG, Wu J, Yu S, Kang KY, Kim ST. Proteasome inhibitors affect appressorium formation and pathogenicity of the rice blast fungus, Magnaporthe oryzae. Plant Pathol J. 2011;27(3):225-31. https://doi.org/10. 5423/PPJ.2011.27.3.225.

Wang RJ, Peng J, Li QX, Peng YL. Phosphorylation-mediated regulatory networks in mycelia of Pyricularia oryzae revealed by phosphoproteomic analyses. Mol Cell Proteomics. 2017;16(9):1669-82. https://doi.org/10. 1074/mcp.M116.066670.

Wang Z, Zhang H, Liu C, Xing J, Chen XL. A deubiquitinating enzyme Ubp14 is required for development, stress response, nutrient utilization, and pathogenesis of Magnaporthe oryzae. Front Microbiol. 2018;9:769. https:// doi.org/10.3389/fmicb.2018.00769.

Wang L, Cai X, Xing J, Liu C, Hendy A, Chen XL. URM1-mediated ubiquitin-like modification is required for oxidative stress adaptation during infection of the rice blast fungus. Front Microbiol. 2019a;10:2039. https://doi.org/ 10.3389/fmicb.2019.02039.

Wang W, Liu N, Gao C, Rui L, Tang D. The Pseudomonas syringae effector AvrPtoB associates with and ubiquitinates Arabidopsis exocyst subunit EXO70B1. Front Plant Sci. 2019b;10:1027. https://doi.org/10.3389/fpls. 2019.01027.

Wilkinson KD. Regulation of ubiquitin-dependent processes by deubiquitinating enzymes. FASEB J. 1997;11(14):1245-56. https://doi.org/10.1096/ fasebj.11.14.9409543.

Wilson RA. Magnaporthe oryzae. Trends Microbiol. 2021;29(7):663-4. https:// doi.org/10.1016/j.tim.2021.03.019.

Wilson RA, Talbot NJ. Under pressure: investigating the biology of plant infection by Magnaporthe oryzae. Nat Rev Microbiol. 2009;7(3):185-95. https:// doi.org/10.1038/nrmicro2032

Xu P, Duong DM, Seyfried NT, Cheng D, Xie Y, Robert J, et al. Quantitative proteomics reveals the function of unconventional ubiquitin chains in proteasomal degradation. Cell. 2009;137(1):133-45. https://doi.org/10. 1016/j.cell.2009.01.041.

Xu N, Luo X, Wu W, Xing Y, Liang Y, Liu Y, et al. A plant lectin receptor-like kinase phosphorylates the bacterial effector AvrPtoB to dampen its virulence in Arabidopsis. Mol Plant. 2020;13(10):1499-512. https://doi.org/10.1016/j. molp.2020.09.016.

Yang J, Chen D, Matar KAO, Zheng T, Zhao Q, Xie Y, et al. The deubiquitinating enzyme MoUbp8 is required for infection-related development, 
pathogenicity, and carbon catabolite repression in Magnaporthe oryzae. Appl Microbiol Biotechnol. 2020;104(11):5081-94. https://doi.org/10. 1007/s00253-020-10572-5.

Ye Y, Rape M. Building ubiquitin chains: E2 enzymes at work. Nat Rev Mol Cell Biol. 2009;10(11):755-64. https://doi.org/10.1038/nrm2780.

Yu F, Wu Y, Xie Q. Ubiquitin-proteasome system in ABA signaling: from perception to action. Mol Plant. 2016;9(1):21-33. https://doi.org/10.1016/j.molp. 2015.09.015.

Zhang L, Yu Z, Xu Y, Yu M, Ren Y, Zhang S, et al. Regulation of the stability and ABA import activity of NRT1.2/NPF4.6 by CEPR2-mediated phosphorylation in Arabidopsis. Mol Plant. 2021;14(4):633-46. https://doi.org/10. 1016/j.molp.2021.01.009.

Zheng Y, Schumaker KS, Guo Y. Sumoylation of transcription factor MYB30 by the small ubiquitin-like modifier E3 ligase SIZ1 mediates abscisic acid response in Arabidopsis thaliana. Proc Natl Acad Sci USA. 2012;109(31):12822-7. https://doi.org/10.1073/pnas.1202630109.

Zheng T, Li Y, Lei W, Qiao K, Liu B, Zhang D, et al. SUMO E3 ligase SIZ1 stabilizes MYB75 to regulate anthocyanin accumulation under high light conditions in Arabidopsis. Plant Sci. 2020;292: 110355. https://doi.org/10.1016/j. plantsci.2019.110355.

\section{Publisher's Note}

Springer Nature remains neutral with regard to jurisdictional claims in published maps and institutional affiliations.

- fast, convenient online submission

- thorough peer review by experienced researchers in your field

- rapid publication on acceptance

- support for research data, including large and complex data types

- gold Open Access which fosters wider collaboration and increased citations

- maximum visibility for your research: over 100M website views per year

At BMC, research is always in progress.

Learn more biomedcentral.com/submissions 